\title{
Portfólio digital desenvolvido a partir da percepção do preceptor cirurgião dentista: um estudo fenomenológico
}

\section{Digital portfolio developed from the perception of the dentist preceptor: a phenomenological study}

Portafolio digital desarrollado a partir de la percepción del preceptor dentista: un estudio fenomenológico Mônica Moura da Silveira Lima ${ }^{1}$, Rose Mary Costa Rosa Andrade Silva ${ }^{2}$, Eliane Ramos Pereira ${ }^{3}$,
Rita de Cássia Ferreira da Silva ${ }^{4}$

Como citar esse artigo. Lima, MMS; Silva, RMCRA; Pereira, ER; da Silva, RCF. Portfólio digital desenvolvido a partir da percepção do preceptor cirurgião dentista: um estudo fenomenológico. Revista Pró-UniverSUS. 2019 Jul./Dez.; 10 (2): 80-82.

\section{Resumo}

O presente estudo tem como objetivo geral compreender o processo de trabalho do preceptor cirurgião dentista, através da avaliação da sua percepçãoexplícita em Maurice Merleau-Ponty, a partir da análise fenomenológica de suas vivências e expertises,na intenção de melhor integrar o serviço-universidade econtribuição da qualidade do ensino na saúde e na assistência da população. Realizar o desenvolvimento de um portfólio digital, a fim de contribuir para o aperfeiçoamento de todos os envolvidos na atividade de preceptoria,também a relação da Universidade e preceptor, possibilitar ao acadêmico obtermelhor conhecimento da realidade no atendimento da população. A metodologia proposta é de cunho descritivo, com abordagem qualitativa. A coleta de dados apresentará um questionário, e observação sistemática.Como população do estudo, os cirurgiõesdentistas preceptores que atuem no serviço de emergência.

Palavras-chave: Preceptor, Odontólogos, Serviços Médicos de Emergência, Integração Docente Assistencial.

\begin{abstract}
Objectives: The present study aims to understand the work process of the preceptor dentist surgeon, through the evaluation of his explicit perception in Maurice Merleau-Ponty, from the phenomenological analysis of his experiences and experiences, in order to better integrate the university service and contribution of the quality of education in health and care of the population. Carry out the development of a digital portfolio, in order to contribute to the improvement of all involved in the preceptorship activity, also the relationship between the University and preceptor, enabling the academic to obtain a better knowledge of the reality in the care of the population. The proposed methodology is descriptive, with a qualitative approach. The data collection will present a questionnaire, and systematic observation. As the study population, the preceptive dental surgeons who work in the emergency service.
\end{abstract}

Keywords: Preceptor, Dentists, Emergency Medical Services, Teaching Assistance Integration.

\section{Resumen}

Objetivos: El presente estudio tiene como objetivo comprender el proceso de trabajo del cirujano dentista preceptor, a través de la evaluación de su percepción explícita en Maurice Merleau-Ponty, a partir del análisis fenomenológico de sus experiencias y experiencias, para integrar mejor el servicio universitario. y contribución de la calidad de la educación en salud y atención de la población. Llevar a cabo el desarrollo de una cartera digital, con el fin de contribuir a la mejora de todos los involucrados en la actividad de preceptoría, también la relación entre la Universidad y el preceptor, permitiendo al académico obtener un mejor conocimiento de la realidad en el cuidado de la población. La metodología propuesta es descriptiva, con un enfoque cualitativo. La recopilación de datos presentará un cuestionario y una observación sistemática. Como la población de estudio, los cirujanos dentales preceptivos que trabajan en el servicio de emergencia.

Palabras clave: Preceptor, Dentistas, Servicios Médicos de Emergencia, Integración de Asistencia Docente.

Afiliação dos autores: 1. Cirurgiã-Dentista. Mestranda do programa de Mestrado Profissional em Ensino na Saúde/MPES, UFF, RJ, Brasil. Email: monicam.silveira@gmail.com ORCID: https://orcid.org/0000-0003-3540-6679

2. Enfermeira. Pós-doutora em Filosofia. Professora Associada da Escola de Enfermagem Aurora de Afonso Costa (UFF). E-mail: roserosauff@gmail.com ORCID: https://orcid. org/0000-0002-6403-2349

3. Enfermeira.Pós-doutora em Enfermagem. Professora Associada da Escola de Enfermagem Aurora de Afonso Costa (UFF). E-mail: elianeramos.uff@gmail.com ORCID: https:// orcid.org/0000-0002-6381-3979

4. Mestranda do programa de Mestrado Profissional em Ensino na Saúde/MPES, UFF, RJ, Brasil. Email:rdecassiaferreira@hotmail.com ORCID: https://orcid.org/0000-0002-81556910 


\section{Introdução}

A formação do Profissional de saúde, traz o desafio de buscar o rompimento de um modelo fragmentado e médico-centrado.A preceptoria trata-se da atuação dos profissionais vinculados aos serviços do Sistema Único de Saúde (SUS), que recebem estudantes, a fim de orientá-los na prática de seu trabalho, em busca da integralidade na formação profissional,e na articulação de melhorias entre trabalho e educação.

As parcerias entre a instituição de ensino e os preceptores, revelam dificuldades no conhecimento do conteúdo teórico apresentado aos estudantes universitários, tendo em vista que a realidade da prática se mostra de forma diferenciada.A técnica adquiridano campo prático deveria caminhar com a teoria, para maior coerência na formação dos acadêmicos, onde o papel do preceptor no processo de aprendizagem se mostrará eficiente.

As vivências dos profissionais que desempenham, tanto função do cuidado, quanto a atribuição de transmitir seus conhecimentos práticos aos futuros profissionais, levamà angústia que devem ser analisadas, para buscar um melhor aprendizado para o acadêmico, e condições satisfatórias de trabalho para o preceptor.

O desenvolvimento de um portfólio digital, que proporcione uma integração do conteúdo didático apresentado ao acadêmico de odontologia.A vivência do preceptor cirurgião-dentista no ambiente de estágio, traz contribuições para investigação na saúde, além do desenvolvimento tecnológico na saúde.

A carência de políticas que trabalhem a temática da percepção docirurgião-dentista preceptor no ambiente hospitalar, dificulta a definição do seu papel na formação do acadêmico de odontologia.Contudo, o presente trabalho pretende contribuir para o aperfeiçoamento de todos os envolvidos na atividade de preceptoria e do ensino na odontologia.

O presente estudo tem como objetivo geral compreender o processo de trabalho do preceptor cirurgião dentista, através da avaliação da sua percepção explícita em Maurice Merleau-Ponty, a partir da análise fenomenológica de suas vivências e expertises, na intenção de melhor integrar o serviço-universidade e contribuição da qualidade do ensino na saúde e na assistência da população.

Desenvolver um portfólio digital como instrumento de práticas assistenciais e ensino, a partir da análise dos dados obtidos na compreensão fenomenológica,na melhoria do processo de formação dos acadêmicos de odontologia e ensinoprático, na intenção de revelar a realidade dos serviços sem desvincular do contexto apresentado em sala de aula.

\section{Materiais e Métodos}

A metodologia proposta é descritiva, com abordagem qualitativa, que visa descrever características vivenciadas por uma população de cirurgiões-dentistas preceptores que atuem no serviço de emergência.Para a coleta de dados, será usado técnicas padronizadas, como questionários e observação sistemática.

\section{Resultados Esperados}

A expectativa da pesquisa, é melhorar a integração da Universidade com os profissionais da preceptoria de odontologia,e levar clareza quanto ao conteúdo didático, objetivos e integração teórico-prático dos discentes, assim, uma melhor adequação do conteúdo teórico passado em sala de aula, em conformidade com a realidade vivida no atendimento do serviço público de emergência.

O estudo pretende ainda impactar positivamente na educação, através da troca de saberes entre o profissional preceptor e o docente, contribuir para a melhor compreensão da realidade e desafios enfrentados em um hospital público, para que ambos consigam buscar soluções para os problemas de saúde,melhorar a qualidade do ensino, assistência da população e responsabilidade social.

\section{Produto}

O presente trabalho de pesquisa, pretende gerar como produto, um portfólio digital, onde se possa integrar o conteúdo didático apresentado ao discente e o preceptor de odontologia, para que a técnica apresentada em sala de aula seja de domínio do mesmo, a proporcionar um aprendizado que faça sentido ao acadêmico.

O portfólio digital desenvolvido a partir da percepção dos preceptores de odontologia, pretende contribuir com o desenvolvimento tecnológico na área de saúde, inovar no ensino, através de um acesso rápido, proporcionar uma melhor relação do ensino universitário, e a prática no campo de estágio.

O conhecimento de novas técnicas, ou até mesmo uma nova abordagemjá dominada pelo preceptor, pode servir como ampliação e melhoria da qualificação do conhecimento, atualização de conteúdos teóricos, com melhor acesso ao conteúdo didático e proporcionar o aprimoramento do ensino, na troca de experiências.

O portfólio pretende informar ao docente, sobre a realidade do ambiente hospitalar, no que diz respeito a seus equipamentos e insumos, para que se possa aplicar a teoria apresentada ao discente no campo de estágio, de modo que a mesma faça sentido e caminhe de maneira harmônica, para impactar positivamente no aprendizado acadêmico, no atendimento da população, 
com responsabilidade social e bem estar de todos.

\section{Referências}

1. Barreto VHL; Monteiro ROS; Magalhães GSG; Almeida RCC; Souza LN. Papel do preceptor da Atenção Primária à Saúde em saúde da formação da graduação e pós-graduação da Universidade Federal de Pernambuco: um termo de referência. Rev. Bras. Educ Méd. 2011; 35(4): 578-583.

2. Brasil. Ministério da Saúde. Secretaria de Atenção à Saúde. Departamento de Atenção Básica. Diretrizes da Política Nacional de Saúde Bucal. Brasília: Ministério da Saúde; 2004.

3. Costa ICC; Araújo MNT. Definição do perfil de competências em saúde coletiva a partir da experiência de cirurgiões-dentistas atuantes no serviço público. Cien Saude Colet. 2011; 16 (Supl. 1): 1181-1189.

4. Luz GW; Toassi RFC. Percepções sobre o preceptor cirurgião-dentista da Atenção Primária à Saúde no ensino da Odontologia. Revista da Abeno. 2016; 16(1): 2-12. doi: 10.30979/rev.abeno.v16i1.210.

5. Matthews, ERIC. Compreender Merleau-Ponty.Petrópolis, RJ; $2^{\mathrm{a}}$ ed., Vozes; $2010 \quad 205 p$.

6. Vasconcelos ACF; Stedefeldt E; Frutuoso MFP. Uma experiência de integração ensino-serviço e a mudança de práticas profissionais: com a palavra, os profissionais de saúde. Interface (Botucatu). 2016; 20(56):14758 